\title{
A 'symptom-triggered' approach to alcohol withdrawal management
}

Jay Murdoch and Janet Marsden

Jay Murdoch is Lead Nurse for Alcohol Liaison, Pennine Acute Hospitals NHSTrust, Manchester; Janet Marsden is Professor of Ophthalmology and Emergency Care, Manchester Metropolitan University Accepted for publication: February 2014

In acute hospital settings, alcohol withdrawal often causes significant management problems and complicates a wide variety of concurrent conditions, placing a huge burden on the NHS. A significant number of critical incidents around patients who were undergoing detoxification in a general hospital setting led to the need for a project to implement and evaluate an evidence-based approach to the management of alcohol detoxification-a project that included a pre-intervention case note audit, the implementation of an evidence-based symptom-triggered detoxification protocol, and a postintervention case note audit. This change in practice resulted in an average reduction of almost $60 \%$ in length of hospital stay and a $66 \%$ reduction in the amount of chlordiazepoxide used in detoxification, as well as highlighting that $10 \%$ of the sample group did not display any signs of withdrawal and did not require any medication. Even with these reductions, no patient postintervention developed any severe signs of withdrawal phenomena, such as seizures or delirium tremens. The savings to the trust (The Pennine Acute Hospital Trust) are obvious, but the development of a consistent, quality service will lead to fewer long-term negative effects for patients that can be caused by detoxification. This work is a project evaluation of a locally implemented strategy, which, it was hypothesised, would improve care by providing an individualised treatment plan for the management of alcohol withdrawal symptoms.

Key words: Detoxification a Alcohol a Chlordiazepoxide a Symptom-triggered approach

Alcohol abuse and dependence represents a very serious health problem worldwide with major social, interpersonal and legal

consequences (Amato et al, 2010). Despite an increasing awareness of its dangers among both the media and the medical profession, alcohol remains a serious and growing public health concern in the UK (Owens et al, 2005). According to Alcohol Concern (2010), the number of dependent drinkers in England now stands at 1.6 million. The number of alcohol- related admissions in England is about one million per year and has been steadily rising (Perkins and Hennessey, 2014), an increase of $100 \%$ since 2002/3 (Alcohol Concern, 2010).

Alcohol Concern states that the cost to the NHS is $£ 2.7$ billion every year and is expected to continue to rise to $\mathrm{f3.7}$ billion (Alcohol Concern, 2010). Although alcohol use and abuse are common among general hospital inpatients, many patients are inadequately assessed and treated for alcohol withdrawal (Repper-Delisi et al, 2008).

Alcohol withdrawal syndrome (AWS) is a cluster of symptoms that may develop in alcohol-dependent people after the cessation of (or reduction in) heavy and prolonged alcohol use (American Psychiatric Association (APA), 2000; World Health Organization (WHO), 2010). AWS inevitably occurs as alcohol consumption ceases on admission to hospital, whether specifically for detoxification ('detox') or as a consequence of admission for other problems.

This project arose from the trust's alcohol specialist nurse's perception that the team tended to be approached to consult on alcohol detoxification only when things went slightly 
'wrong' such as when a patient's symptoms exacerbated and he/she became difficult and timeconsuming to manage. They were worried about a lack of education in all clinicians about detoxification and a lack of evidence-based protocols to assist in patient care. A significant number of critical incidents involving patients who were undergoing detoxification added to the need for a project to implement and evaluate evidence-based approaches to the management of alcohol detoxification. This project was developed to standardise alcohol detoxification regimes across a single-site hospital due to perceptions that existing regimes were not evidence-based and were not consistent.

\section{AWS}

The clinical presentation of AWS varies from mild to serious. The onset of symptoms typically occurs up to 48 hours after the last consumption of alcohol, and up to 72 hours if delirium tremens are considered (Amato et al, 2010). Delirium tremens occurs in only about 5\% of patients undergoing alcohol withdrawal, but has the highest mortality rate: 5-20\% in inappropriately managed patients. It is a medical emergency and is an advanced state of autonomic hyperactivity. There is substantial individual variation in the clinical manifestations of alcohol withdrawal, ranging from very mild tremor and irritability to significant autonomic hyperactivity (e.g. sweating or pulse rate greater than 100 beats per minute (APA, 2000; Wetterling et al, 2001; Amato et al, 2010). The Diagnostic and Statistical Manual of Mental Disorders (DSM-

IV) (APA, 2000) continues the symptom list with the following symptoms: tremor, insomnia, nausea or vomiting, transient visual, tactile or auditory hallucinations or illusions, psychomotor agitation, anxiety and seizures. To define AWS, the patient must have two or more of these symptoms following cessation or reduction of alcohol (McKeon, 2008).

The relation between alcohol intoxication, dependence, tolerance and withdrawal is not fully understood. But we do know that there is a clear relation between alcohol and changes

\section{Development of the tool, education and implementation}

Withdrawal severity varies greatly and the amount of medication needed to control symptoms can also vary significantly. Chlordiazepoxide is typically used in practice (NICE, 2010b), and was therefore the drug of choice for the protocol, though diazepam and lorazepam can be equally efficacious (Asplund et al, 2004). Chlordiazepoxide is usually administered in predetermined prophylactic dosing schedules varying between 3 and 10 days (Daeppen et al, 2002; McGregor et al, 2003; Saitz, 2004; McKinley, 2005; NICE, 2010b).

Although this method is used frequently throughout hospitals, predetermined fixed dosing regimens may subject many patients to unnecessary medication, over-sedation and longer hospital stays (Asplund et al, 2004; Saitz et al, 2004; Hardern and Page, 2005; NICE, 2010a; NICE, 2010b). Saitz et al (2004)

state that many patients can safely undergo withdrawal from alcohol comfortably and without pharmacological intervention, which was seen in the results of this project.

It is clear, then, that a more individualised approach to management would benefit patients. This was achieved using a symptom- triggered therapy protocol, consisting of monitoring patients and providing medication only when symptoms of alcohol withdrawal developed. Symptoms were identified with a validated assessment tool, the revised Clinical Institute of Withdrawal Assessment Scale (CIWA-ar) (McKeon, 2008). The symptom- triggered approach has demonstrable success (Daeppen et al, 2002; Day et al, 2004; Hardern and Page, 2005) and is recommended by NICE (2010a). The CIWA-ar scores the severity of nausea, sweating, agitation, headache, anxiety, tremor, sensory disturbances and orientation, and is easy to use (McKeon, 2008). Repeated scoring at suitable 
intervals monitors the response to treatment and helps determine if further pharmacotherapy is needed.

These tools were integrated into a single booklet, including a policy for detoxification and an integrated care pathway (ICP) in which nurses were enabled to administer chlordiazepoxide, with the dose dependent on the symptom score. A steering group engaged with hospital directorates and gained approval. Plans for hospital-wide implementation were put into place, which included email alerts to all consultants, alcohol link nurses and allied health professionals. There were also advertising posters, and articles in trust magazines and on notice boards. Before implementation,

80 hour-long, separate teaching sessions were delivered by the alcohol team over a 2-week period to all appropriate clinical staff. Implementation followed, assisted by a timely changeover of junior doctors and by constant encouragement and endorsement of the ICP by the alcohol team Post-intervention audit

A post-intervention audit took place 4 months after the implementation of the policy and ICP. The same method and audit tool were used to obtain data. The sample group was the same size as the initial sample, recruited as consecutive patients and fulfilling the same criteria. A staff-satisfaction questionnaire was also used to gain insight into clinicians' thoughts on the new policy and ICP. A mix of open and closed questions, sent to 300 clinical staff, obtained qualitative findings.

\section{Results}

Results of pre-implementation audit The average number of days to detox a patient was 6.36 . On average it costs $£ 300$ per night for an inpatient stay, making the average cost of these detox attempts f1908. The average dose of chlordiazepoxide to complete an inpatient detox was $563.3 \mathrm{mg}$. Forty percent of patients were given PRN (when required) medication, but in only $10 \%$ of these (two patients) was the reason documented. Six of the prescriptions did not usechlordiazepoxide but other medication.

There were a number of prescribing errors, such as:

- Dosing regimens prescribed in the wrong area of prescription chart

- No maximal dose entered on chart

- Tapering doses that increased instead of decreased

- Prescriptions that had fixed doses for a number of days instead of decreasing

- Only PRN medication written, with no guidance on administration

- Four prescriptions had fixed dosing, so there was no reduction, which is needed in withdrawal treatment.

There was great variation in the prescribing methods used when reducing chlordiazepoxide and in the number of days to complete a detox (4 to 10). Most importantly, a total of 11 patients developed signs of severe AWS: 5 developed delirium tremens and 6 had post- admission seizures.

Results of post-implementation audit The average number of days to complete detox after implementation of the ICP was

2.48. On average it costs $£ 300$ per night for an inpatient stay, making the average cost of these detox attempts $f 744$. The average dose of chlordiazepoxide to complete an inpatient detox was $167.2 \mathrm{mg}$.

Chlordiazepoxide was the only drug used. Five patients required no chlordiazepoxide.

With the CIWA-ar scoring chart, it was clear what symptoms the patient was displaying. The nurse could then administer a dose of chlordiazepoxide as required to alleviate the symptoms. Documentation had improved because now all clinicians were using the same paperwork to manage this patient group. Use of the CIWA-ar also identified exactly what symptoms the patient was displaying. 
Before the project, 11 people from the sample group developed severe signs of AWS such as seizures or delirium tremens. Following the change, no patient developed severe signs of AWS. Clearly, this is only for this sample and a larger study would have to be done to ensure this finding was accurate. However, in light of the theory behind the symptom-triggered approach, identifying symptoms early should prevent exacerbation.

All prescriptions were written correctly and the dose of administration was only wrong on three occasions, with patients not having the right amount in accordance with their CIWA-ar score.This was put down to nursing error, as the flow chart had not been followed correctly and this triggered further educational input (refresher sessions for individual nurses were given where needed). No patients from this sample group developed signs of delirium or seizures after starting the ICP.

A total of 212 questionnaires were returned by a full range of clinical staff, from consultant to staff nurse, $97 \%$ of whom believed that the ICP was user-friendly.The vast majority (98\%) believed that there had been improvements in care for this patient group, and that detox was achieved much quicker and was of benefit both to them (through reduced workload) and to the patients themselves. From the questionnaires, the project team concluded that clinicians were satisfied with the change, mostly because of the benefits for patients. The results identified that an increase in satisfaction correlated directly with the increase in compliance.

It was hypothesised at the start of the project that the use of an evidence-based, symptomtriggered, individualised approach to withdrawal management, if used in an acute setting, would improve patient care. It was thought that the amount of chlordiazepoxide administered would decrease, resulting in a shorter treatment duration. These results were confirmed by the Mann-Whitney nonparametric statistical test. This test was chosen because there was no specific distribution for the population, resulting in skewed data. The statistics were analysed with STATA software and resulted in a $p$ value of $0.001 \quad(p=0.001)$. This was the case for both duration of treatment and the amount of chlordiazepoxide administered, thereby confirming the hypothesis.

\section{Discussion}

The results of the pre-and post-implementation audit accords with findings from previous researchers on this subject (Sullivan et al, 1989; Daeppen et al, 2002; Day et al, 2004; Saitz et al, 2004). The samples were not case-controlled, which some might argue undermines the conclusions. Yet the results are too startling to be the result of coincidence (the statistical analysis showed that the likelihood of them being a coincidence is just $0.01 \%$ ).

Figure 5 shows that the time for a patient to complete a detox has been greatly reduced since the implementation of the ICP. Furthermore, the amount of chlordiazepoxide needed to complete a detox has also fallen significantly (Figure 6).

Even though significantly less chlordiazepoxide was required in the majority of cases to manage AWS, not one person from this sample group developed severe signs of withdrawal. It is most likely that this is due to the patients being assessed more frequently with a validated assessment tool, in conjunction with staff having an increased knowledge of the withdrawal process. It also provides an individualised treatment plan. The fact that signs and symptoms were being detected earlier ensured that patients were adequately medicated and autonomic responses were kept to a minimum, safeguarding against the development of severe AWS.

As a contained booklet, it was clear that the ICP was allowing staff to ensure documentation was correct and acting as a guide to guarantee that staff knew exactly when to assess and manage the level of withdrawal they were presented with. Documentation as a whole had improved. The CIWA-ar score meant that staff identified individual symptoms of withdrawal instead of using broad terminolgy and grouping. 
If these results are translated into even very rough costings, the savings to the trust are obvious. Pre-implementation, the average cost of an inpatient stay for detoxification was $f 1908$. Postimplementation, this fell to

f744. More importantly, fewer drugs were used.These drugs were appropriate in all cases and no severe symptoms occured in any of the patients from the audit sample.

Implementation was not without its problems, however, and a key stage was the arrival of new doctors in the trust, which really moved the change forward. The policy was not a change in practice for these new doctors, who accepted it as trust policy and began to use it.To an extent, then, they drove the rest of the clinical team to use the ICP and move the care of this patient group forward.

From the point of view of the alcohol team, which had spent a huge amount of time and energy educating and implementing the policy, the surge in referrals was a validation of its efforts. The increase in compliance meant more patients were being assessed by an alcohol specialist, resulting in in-depth alcohol histories, appropriate treatment options, prompter discharges and, when required, more successful detox attempts. The alcohol team was also identifying trends: for example, it noted early on that not all patients who were alcohol-dependent showed physical signs of withdrawal and required medication to stop. Before moving to the symptom-triggered approach to management, patients would have automatically been started on a prophylactic detox regime and could be in hospital for up to 10 days to complete it, even though their bodies did not require any medication. Some people were completing detox in as little as 2 days.

Both these last points showed nurses that, if looked after appropriately, the patients required less management overall and spent less time in hospital. The alcohol team was also getting fewer complaints from its patients with regard to treatment, due to their detox being managed appropriately. An early referral to the alcohol team showed patients that the hospital was trying to help themtheir stay was much more than just having pills four times a day and they had proper treatment plans and aftercare organised.

The team's credibility grew within the hospital due to improved patient care.Clinicians could see that the ICP was indeed improving care, improving documentation, improving patient satisfaction and reducing clinical incidents. These thoughts were reinforced with the results of the post-change audit.

\section{Conclusion}

This project has achieved what it set out to achieve: an evidence-based integrated care pathway for a group of patients for whom care had been less than optimal, resulting in repeated detoxification and poor outcomes. The cost savings, both in terms of inpatient stay and drug use, are quantifiable. The potential benefits of fewer repeat episodes of detoxification and the revolving-door effect, as well as the greater patient satisfaction, are less easy to quantify, but doing so remains an aim for future work.

Excessive drinking is currently the second greatest risk to public health in developed countries (Kaner, 2010). Although most of that risk is avoidable, the risk is clearly apparent with the large number of hospital admissions every year. As many as one in three A\&E attendees have consumed alcohol immediately before their presentation and more than two in three entries after midnight may be alcohol related (Williams et al, 2005).

The focus on alcohol is becoming more apparent. Initiatives such as the Commissioning for Quality and Innovation (CQUIN) payment framework enable commissioners to reward excellence by linking a proportion of healthcare income to the achievement of the local quality improvement goals. The fact that alcohol is such a major problem for patients, but is also now linked to financial gain for trusts, makes it even more imperative that treatment is timely and effective. This project demonstrates one such method. 
Reference

Alcohol Concern (2010) Investing in Alcohol Treatment - Reducing Costs and Improving Lives. Alcohol Concern, London

Amato L, Minozzi S, Vecchi S, Davoli M (2010) Benzodiazepines for alcohol withdrawal. Cochrane Database of Systematic Reviews, Issue 3.Art. No:CD005063. DOI: 10.1002/14651858.CD005063.pub3

American Psychiatric Association (2000) Diagnostic and Statistical Manual of Mental Disorders. 4th edn. American Psychiatric Association. Washington DC, USA

Asplund CA,Aaronson JW,Aaronson HE (2004) 3 regimens for alcohol withdrawal and detoxification. J Fam Pract 53(7): 545-54

Daeppen JB, Gach P, Ulrika L, et al (2002) Symptom-triggered vs fixed-schedule doses of benzodiazepine for alcohol withdrawal. Arch Intern Med 162(10): 1117-21

Day E, Patel J, Georgiou G (2004) Evaluation of a symptom- triggered front-loading detoxification technique for alcohol dependence: a pilot study. Psychiatric Bulletin 28: 407-10

Gold MS, Aronson MD (2011) Alcohol and abuse and dependence: epidemiology, clinical manifestations, and diagnosis. UpToDate Version 19(2). http://tinyurl.com/ ozluvzu (accessed 14 February 2014)

Hardern R, Page AV (2005) An audit of symptom-triggered chlordiazepoxide treatment of alcohol withdrawal on a medical admissions unit. Emerg Med J 22(11): 805-6

Hoffman PL, Tabakoff B (1996) Alcohol dependence: a commentary on mechanisms. Alcohol Alcohol 31(4): 333-40

Kaner E (2010) Brief interventions against excessive alcohol consumption. In: Oxford Textbook of Medicine. Oxford University Press, Oxford

McGregor C, Machan A, White J (2003) In-patient benzodiazepine withdrawal: comparison of fixed and symptom-triggered taper methods. Drug Alcohol Rev 22(2): 175-80

McKeon A (2008) The alcohol withdrawal syndrome. J Neurol Neurosurg Psychiatry 79(8): 854-62 McKinley MG (2005) Alcohol withdrawal syndrome overlooked and mismanaged? Crit Care Nurse 25(3): 40-8 National Institute for Health and Care Excellence (2010a) Alcohol use disorders: Diagnosis and clinical management of alcohol related physical complications. CG100. NICE, London National Institute for Health aCare Excellence (2010b)

Alcohol use disorders: sample chlordiazepoxide dosing regimens for use in managing alcohol withdrawal. CG115. NICE, London

Owens L, Gilmore IT, Pirmohamed M (2005) How do NHS general hospitals in England deal with patients with alcohol-related problems? A questionnaire survey. Alcohol Alcohol 40(5): 409-12

Perkins C, Hennessey M (2014) Understanding alcohol- related hospital admissions. Blog Public Health Matters. Public Health England. http://tinyurl.com/mrmxa6l (accessed 14 February 2014)

Repper-Delisi J, Stern TA, Mitchell M, et al (2008) Successful implementation of an alcohol-withdrawal pathway in a general hospital. Psychosomatics 49(4): 292-9. doi: 10.1176/appi.psy.49.4.292.

Saitz R, Mayo-Smith MF, Roberts MS (2004) Individualised treatment for alcohol withdrawal: a randomized double blind controlled trial. JAMA 272(7):519-23

Sullivan JT, Sykora K, Schneiderman J, et al (1989) Assessment of alcohol withdrawal: the revised clinical institute withdrawal assessment for alcohol scale (CIWA-ar). Br J Addict 84(11): 1353-7

Wetterling T, Driessen M, Kanitz RD, Junghanns K (2001) The severity of alcohol withdrawal is not age dependent. AlcoholAlcohol36(1):75-8 


\section{Williams S, Brown A, Patton R, et al (2005) The half-life of the 'teachable moment' for alcohol misusing patients in the emergency department. Drug Alcohol Depend 77(2): 205-8 World Health Organization (2010) International Statistical Classification of Diseases and Related Health Problems 10th Revision.}

\section{Key Points}

Despite an increasing awareness of its dangers alcohol remains a serious and growing public health concern in the UK

- Alcohol-related admissions are steadily rising and although alcohol misuse is common among general inpatients many are inadequately assessed and treated for alcohol withdrawal

- This project looked at ways of improving the management of patients withdrawing from alcohol and changed practice

- A 'symptom-triggered' approach to managing withdrawal symptoms in an acute hospital setting is achievable and can improve outcomes $\mathrm{f}$ patients, and reduce treatment duration and the amount of medication used

- Alcohol misuse should be a high priority on every hospital trust's agenda-projects such as this one can improve patient experience and st awareness

\footnotetext{
1. Audit tool and rationale for questions

as the alcohol ICP used in the management of this patient? portant to identify if the trust guidelines are being met 
in neurotransmissions in the brain (McKinley, 2005). The chronic presence of alcohol on the brain leads to neuroadaptation and affects brain receptors because the brain tries to maintain a homeostatic balance (Hoffman and Tabakoff, 1996; Amato et al, 2010). Neurotransmitters transmit information between neurons via synapses, either exciting or inhibiting impulses along the neurons (McKinley, 2005). Alcohol acts as an antagonist at key receptors-some of the key changes involve reduced brain gamma-aminobutyric acid (GABA) levels and GABA-receptor sensitivity (Gold and Aronson, 2011). Prolonged intake causes adjustment of the central nervous system, reducing the initial short-term effects (McKinley, 2005). Abrupt cessation or withdrawal of alcohol intake causes a rebound stimulatory effect, resulting in autonomic hyperactivity, leading to symptoms of AWS (McKinley, 2005).

It is important to treat AWS in order to decrease the severity of symptoms, preventing more severe alcohol withdrawal phenomena such as seizures, delirium tremens and Wernicke's encephalopathy, which can be fatal. The advances in knowledge of neurobiology and neurochemistry have prompted the use of drugs in the treatment of alcohol dependence and withdrawal that will allow the GABA pathways to restore normal brain function (Amato et al, 2010). There is a variety of differing medications that work on the GABA neurotransmitters in the brain to treat AWS

(Amato et al, 2010; National Institute for Health and Care Excellence (NICE), 2010a) and the trust's alcohol steering group decided to develop a protocol based on benzodiazepines, which are widely recognised as the drug of choice for detoxification (Daeppen et al, 2002; Asplund et al, 2004; Saitz et al, 2004; McKinley, 2005; Amato et al, 2010; NICE, 2010a).

Benzodiazepines relieve the symptoms of alcohol withdrawal and reduce the frequency of seizures and delirium tremens (Saitz et al, 2004) by acting on the GABA receptors and thus stopping the autonomic responses that follow cessation of alcohol (Asplund et al, 2004; McKinley, 2005; NICE, 2010a). When

administered appropriately, benzodiazepines will not only prohibit the development of AWS but also normalise brain function (Amato et al, 2010).

insomnia, nausea or vomiting, transient visual, tactile or auditorythe presence of

alcohol tolerance, the presence of an alcohol withdrawal syndrome when drinking has ceased or been reduced (i.e. physical dependence on alcohol), the intake of alcohol to relieve signs of withdrawal, and the presence of difficulties in controlling drinking, with a strong desire or compulsion to drink (Hoffman and Tabakoff 1996).

The number of dependent drinkers in England now stands at 1.6 million (Alcohol Concern 2010). The cost to the NHS is 2.7 billion pounds every year and is expected to continue to rise to 3.7 billion (Alcohol Concern 2010). Although alcohol use and 
abuse are common among general hospital inpatients, many patients are inadequately assessed and treated for alcohol withdrawal (Repper-Delisi 2008). Alcohol Withdrawal Syndrome (AWS) is a cluster of symptoms that may develop in alcohol dependent people after the cessation of (or reduction in) heavy and prolonged alcohol use (American Psychiatric Association 2000). The clinical presentation varies from mild to serious and the onset of symptoms typically appear up to forty-eight hours after the last consumption of alcohol, and actually up to seventy-two hours if we consider the uncommon case of delirium tremens (Amato et al 2011). Delirium tremens happens only in about $5 \%$ of patients undergoing alcohol withdrawal however has the highest mortality rate (5 to $20 \%$ in inappropriately managed patients), it is a medical emergency and is an advanced state of autonomic hyperactivity. There is substantial individual variation in the clinical manifestations of alcohol withdrawal, ranging from very mild tremor and irritability to significant autonomic hyperactivity (e.g. sweating or pulse rate greater than one hundred) (Amato et al 2011, Wetterling et al 2001, Hoffman and Tabakoff 1996). DSM-IV continues the symptom list with the following symptoms; tremor, insomnia, nausea or vomiting, transient visual, tactile or auditory hallucinations or illusions, psychomotor agitation, anxiety and seizures (American Psychiatric Association 2000). To define AWS, the patient must have two or more of these symptoms following cessation or reduction of alcohol (McKeon 2008). In general hospitals, alcohol withdrawal often causes significant management problems and complicates the management of a wide variety of concurrent conditions (Foy et al 1997). This places a huge burden on the National Health Service (NHS). Alcohol Concern's (2011) research showed that the number of hospital admissions was 1.1 million in 2009/2010, a 100\% increase since 2002/03.

The alcohol specialist nurses noted that they tended to be approached to consult on alcohol detoxification only when things went slightly 'wrong' and were worried, about a lack of education in all clinicians around detoxification and a lack of evidence based protocols to assist in patient care. A significant number of critical incidents around patients who were undergoing detoxification added to the led to the need for 
a project to implement and evaluate an evidence based approach to the management of alcohol detoxification.

The Project

The project consisted of three phases

- a pre intervention case note audit looking at the method of detoxification, the drugs used and the time taken

- the development of an evidence based protocol for the management of detoxification along with the education of staff and the implementation of the protocol

- a post intervention case note audit

The pre intervention audit

A sample of fifty patient case notes were analysed in the pre-change audit. It was felt this size would highlight trends in the total population and was a sufficient enough size to extract adequate findings. This was a convenience sample using the first fifty patients who were referred to the alcohol service who had that completed a detox as an inpatient. All patients had to fit the ICD 10 classifications of alcohol dependency.

The findings gathered from this were taken in a quantitative format, this was felt appropriate to make smoother comparisons with the post-change audit to complete the audit cycle. The medical records of these patients were manually searched to obtain the data needed in accordance with the audit tool.

Figure 1

Audit Tool

1. Was the alcohol ICP used in the management of this patient? Important to identify if the Trust guidelines are being met 
2. Number of days to complete detox?

Important to compare pre ICP and post ICP to note any improvement

3. How much Chlordiazepoxide was administered?

Important to compare pre ICP and post ICP to note any improvement

4. If ICP not commenced. Was PRN medication utilised?

This will identify if predetermined dosing regimes are used are staff trying to alleviate further symptoms with as required medication

5. If PRN medication was used was it identified in the notes why?

This will highlight the need for CIWA scoring system that is part of ICP to improve assessment and documentation

6. What drug is used in the detox process?

Will see if we are using the best available pharmacology to manage this group

7. Is there consistency with prescribing?

This will identify any problems with medical prescribing. Are the medicines prescribed in proper place? Are all areas of Kardex complete?

8. Did anyone develop delirium or seizure activity following commencement on a detox?

This will help identify differences between symptom-triggered management and predetermined dosing. 
Development of the tool, education and implementation

The interrelationship between alcohol intoxication, dependence, tolerance and withdrawal are not fully comprehended, but a clear relationship exists between alcohol and alterations in neurotransmissions in the brain (McKinley 2005). The chronic presence of alcohol on the brain leads to neuroadaptation and affects brain receptors due to the brain trying to attempt to maintain a homeostatic balance (Amato et al 2011, Hoffman and Tabakoff 1996). Neurotransmitters transmit information between neurons via synapses. They either excite or inhibit impulses along the neurons (McKinley 2005). Alcohol acts as an antagonist at key receptors, some of the key changes involve reduced brain gamma-aminobutyric acid (GABA) levels and GABA-receptor sensitivity (Gold and Aronson 2011). GABA allows more chloride to enter the neuron, making the depolarisation of neuronal cell membranes less likely thereby inhibiting the cell (McKinley 2005). McKinley (2005) also describes the second important neurotransmitter affected by chronic alcohol abuse that is the excitatory neurotransmitter $N$-methyl-D-aspartate (NMDA). NMDA regulates neuron excitability, increasing depolarisation of the neuronal membrane by regulating the flow of calcium into the neuron. Chronic alcohol misuse results in a decrease in GABA inhibitory function and an increase in NMDA sensitivity (Amato et al 2011). Prolonged intake causes adjustment of the central nervous system (CNS), reducing the initial short term affects (McKinley 2005). Abrupt cessation of intake of alcohol or withdrawal causes a rebound stimulatory effect, resulting in autonomic hyperactivity, leading to symptoms of AWS (McKinley 2005) See (Figure 1.). It is important to treat AWS in order to decrease the severity of symptoms, preventing more severe alcohol withdrawal phenomena such as seizures, delirium tremens and WE, which could prove fatal. The advances in knowledge of neurobiology and neurochemistry have prompted the use of drugs in the treatment of alcohol dependence and withdrawal that will allow the GABA pathways to restore normal brain function (Amato et al 2010). There are a variety of differing medications that work on the GABA neurotransmitters in the brain to treat AWS (Amato et al 2011 and NICE 2010). These drugs include benzodiazepine GABAergic medications, which 
consists of mainly benzodiazepine and non-benzodiazepine, GABAergic compounds, which involve carbamazepine, gabapentin, valproic acid, topiramate, Gamahydroxybutyric acid (GHB), baclofen, flumazenil and many more besides (Amato et al 2011).

It was decided to develop a protocol based on Benzodiazepines that are widely recognised as the drug of choice for detoxification (Saitz et al (2011), Amato et al (2010), NICE (2010), McKinley (2005), Chad et al (2004) and Daeppen et al (2002)).

Benzodiazepines ameliorate the symptoms of alcohol withdrawal and reduce the frequency of seizures and delirium tremens (Saitz et al 2011). Metabolic and cellular changes do not resolve with cessation of drinking (McKinley 2005). Due to the changes in this homeostatic balance of the brain increased autonomic response develops in some patients presenting itself in the form of AWS. This is why Benzodiazepines are required to act on the GABA receptors mirroring the way alcohol does stopping autonomic responses noted following cessation from alcohol (NICE 2010, McKinley 2005 and Chad 2004). When administered appropriately Benzodiazepines will not only prohibit the development of AWS but by working on the GABA receptors will normalise brain function (Amato et al 2010).

Chlordiazepoxide is more typically used in practice (NICE 2010b) was therefore the drug of choice for the protocol however Diazepam and Lorazepam can be equally efficacious (Chad et al 2004).

Although withdrawal severity varies greatly, and the amount of medication needed to control symptoms can also vary significantly Chlordiazepoxide is usually administered in predetermined prophylactic dosing schedules varying between three and ten days (Saitz et al 2011, NICE 2010b, McKinley 2005, Daeppen et al 2002 and McGregor et al 2002). Although this method is used frequently throughout hospitals, predetermined fixed dosing regimes may subject many patients to unnecessary medication, over sedation and longer hospital stays (Saitz et al 2011, NICE 2010, NICE 2010b, Hardern and Page 2005, Chad et al 2004 and McGregor et al 2002). Saitz et al (2011), states that many patients actually undergo alcohol withdrawal safely and comfortably without pharmacologic intervention. 
It is clear then that a more individualised approach to management would benefit patients. Symptom-triggered therapy, which consists of monitoring patients and providing medication only when symptoms of alcohol withdrawal appear is an alternative and could individualise and improve the management of alcohol withdrawal (Saitz et al 2011 and NICE 2010b). A number of studies have demonstrated its successful use (Daeppen et al (2002) Day et al (2004) Hardern and Page 2005, ) and it is recommended by NICE (2010) Symptoms can be identified by the use of a validated assessment tool. A quick and useful assessment tool is the revised Clinical Institute of Withdrawal assessment Scale (CIWA-ar) (McKeon 2008). This ten-item scale scores the severity of nausea, sweating, agitation, headache, anxiety, tremor, sensory disturbances and orientation (McKeon 2008). Sullivan et al (1989) state competent nurses can carry out an evaluation in less than two minutes and the inter-rater reliability is high. Repeated scoring at suitable intervals depending on scoring monitors the response to treatment and helps to determine if further pharmacotherapy is needed.

Using project management methodology and utilising a steering group of stakeholders within the Trust, a policy for detoxification with an integrated care pathway as an integral part of it. Hs was approved by all governing boards of all directorates as patients might be detoxifying in any Trust clinical area and it was crucial that all areas used the policy. This agreement was reached and plans for hospital wide implementation put into place which included email alerts to all consultants, alcohol link nurses, and allied health professionals for cascading to teams as well as advertising posters and articles in Trust magazines and on notice boards. Prior to implementation, eighty, hours long, separate teaching sessions were delivered within a two week period The period after implementation day and the weeks after were spent chasing doctors to change prescriptions from tapering prophylactic doses to the symptom-triggered approach advocated by the policy, training more and more staff, and questioning why nurses who were aware of the Policy were still administering medication the old way. It was clear no matter how rigorous propaganda is there is still a huge proportion of staff who apparently have never seen it or indeed encountered a member of the 
alcohol team, a link nurse or their ward manager in the weeks before its implementation. Over a period of weeks though, the alcohol team noticed a change in practice and more implementation of the policy and ICP and this was helped hugely by the changeover of junior doctors in August. They did not need to change practice, just to be told about Trust policy and this heralded a step change in practice. Doctors began to use the ICP and ask nurses to implement it, as a matter of normal practice.

The post intervention audit

To compete the audit cycle, the author completed a post-change audit four months after the implementation of an alcohol withdrawal policy and ICP. The same method and audit tool was used to obtain data. However the sample group were different patients, although all met the same criteria as pre-change audit and the sample size was the same. A staff satisfaction questionnaire was also be used to gain insight into clinician's thoughts on the new policy and ICP. A mixture of open and closed questions were utilised to gather qualitative findings.

Results of pre implementation audit

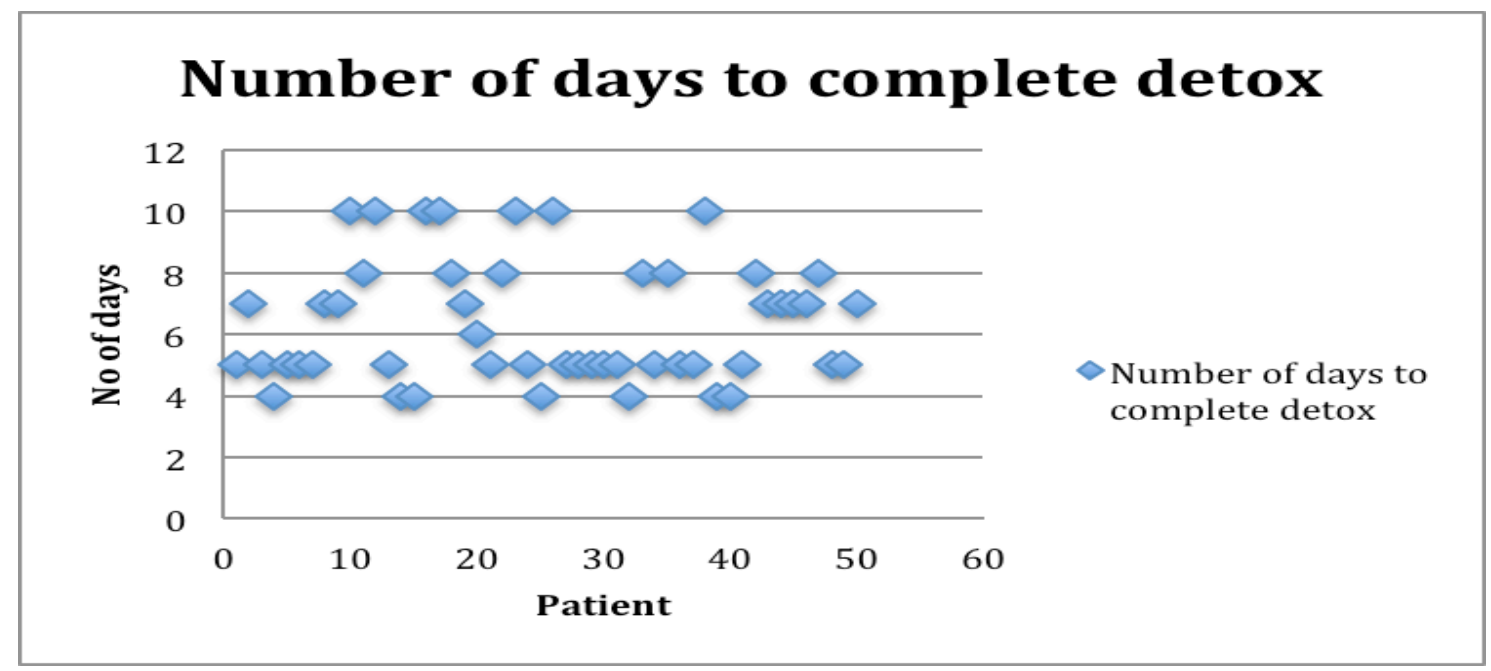


- Average Number of days to detox a patient was 6.36 days.

- On average it costs $£ 300$ per night for inpatient stay therefore the average cost of these detox attempts is $f 1,908$.

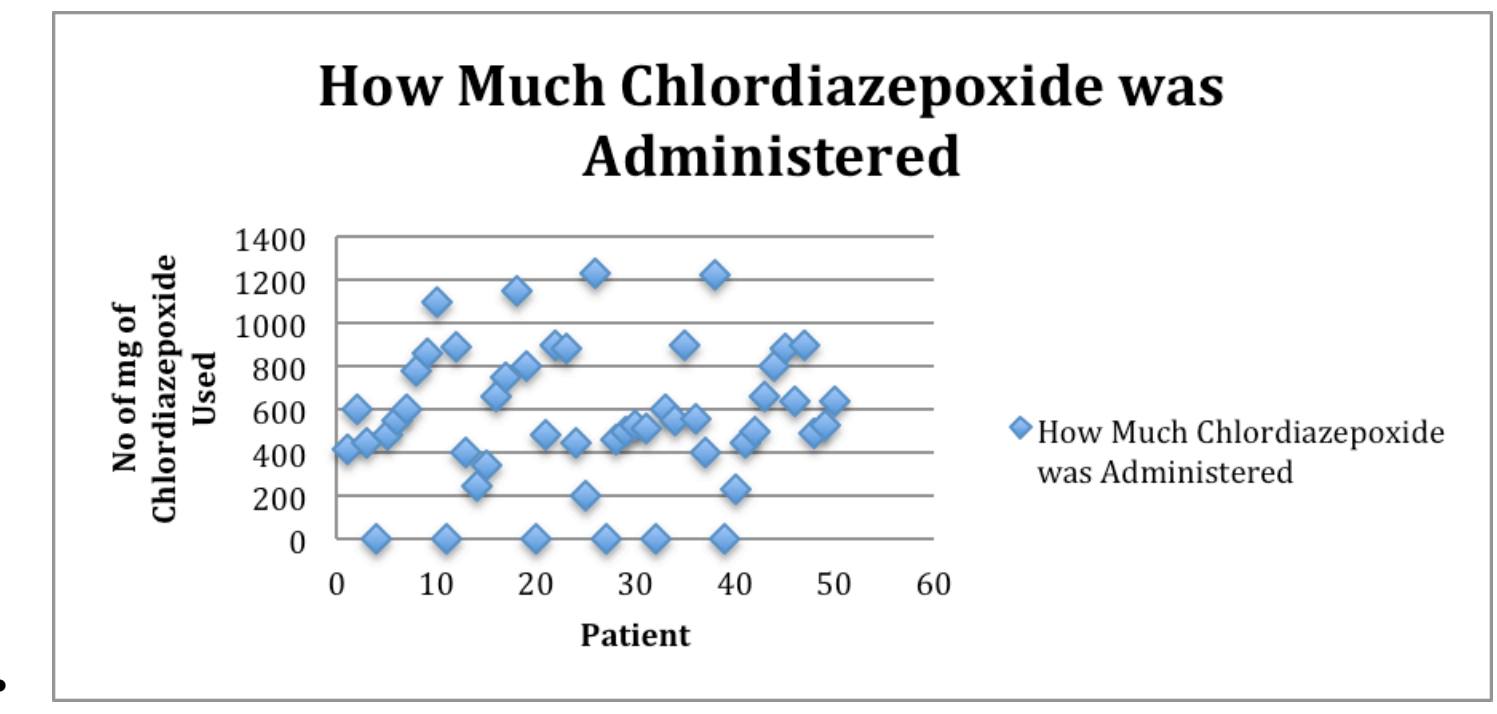

- Average number of milligrams of Chlordiazepoxide to complete an inpatient detox was $563.3 \mathrm{mg}$. $40 \%$ were given PRN medication but in only $10 \%$ of these ( 2 cases) was the reason for this documented

- Six of the prescriptions did not use Chlordiazepoxide but used other medication.

There were a number of prescribing errors made such as: -

- Dosing regimes prescribed in the wrong area of prescription chart

- No maximum dose entered on chart

- Tapering doses that increased instead of reducing

- Prescriptions that had fixed doses for a number of days instead of reducing

- Only PRN medication written with no guidance on administration

- 4 prescriptions had fixed dosing so there was no reduction which is needed in withdrawal treatment

- Large amount of variation noted when reducing Chlordiazepoxide

- Days to complete a detox ranged from four to ten days 
Most importantly, Eleven patients developed signs of severe AWS. Five developed the 'Delirium Tremens' and six had seizures post admission.

Results of post implementation audit

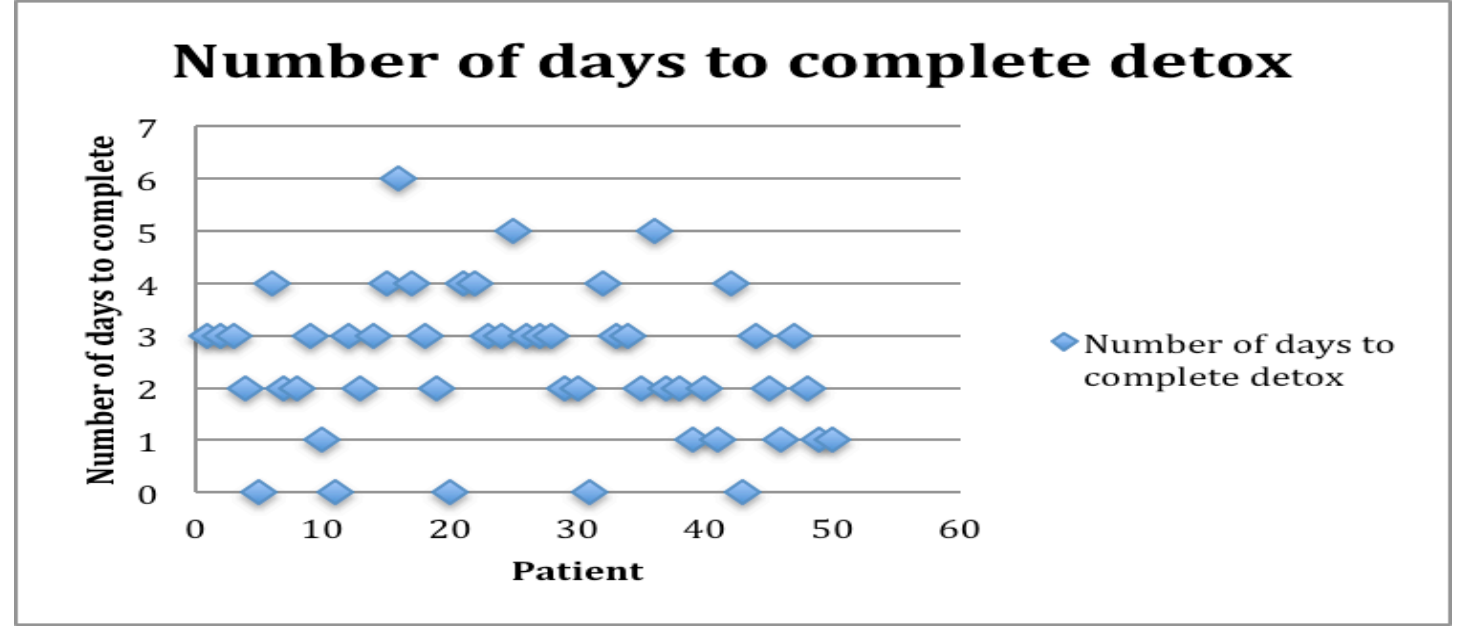

- Average number of days to complete detox is 2.48 days

- On average it costs $£ 300$ per night for inpatient stay therefore the average cost of these detox attempts is $f 744$.

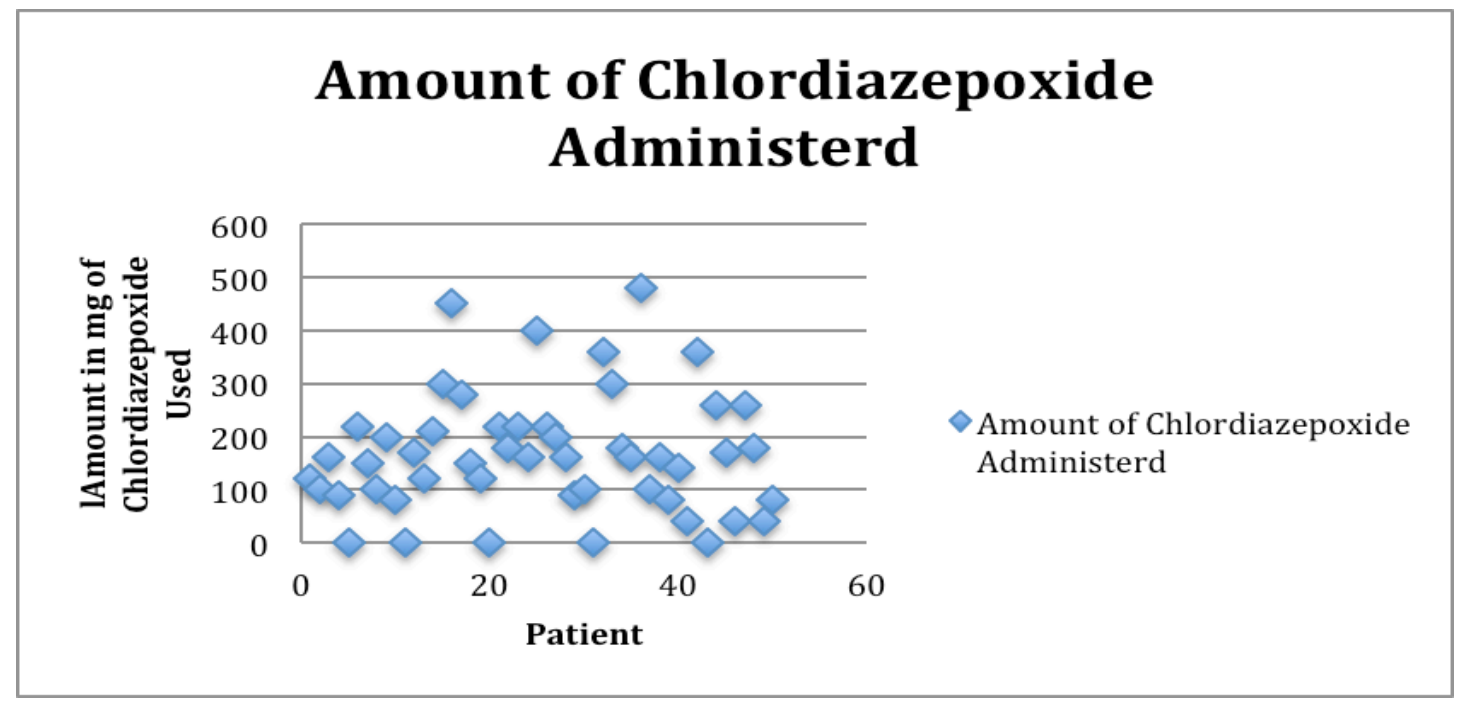

- Average number of milligrams of Chlordiazepoxide to complete an inpatient detox was $167.2 \mathrm{mg}$.

- Chlordiazepoxide was the only drug used 
5 patients required no Chlordiazepoxide

It can be noted that with the use of the CIWA-ar scoring chart it was clear what symptoms the patient was showing. With this the nurse could prescribe X-amount of Chlordiazepoxide as required to alleviate the symptoms. Documentation had improved because now all clinicians were using the same paperwork to manage this patient group.

All prescriptions were written correctly as per ICP. The dose of administration was only wrong on three occasions with patients not having the right amount in accordance to their CIWA-ar score. This was put down to nursing error, as they had not followed the flowchart correctly.

No patients from this sample group developed signs of delirium or seizures post commencement on the ICP.

212 questionnaires, consisting of a mixture of closed ad open ended questions were returned by a full range of staff from consultant to staff nurse, $97 \%$ of whom felt that the ICP was user friendly. 98\% felt that there had been improvements in care for this patient group and felt that detox was no much quicker and was of benefit both to workload and to the patient themselves.

Discussion

The results of the author's audit cycle mirror previous research Daeppen et al (2002), Day et al (2004),Harden and Page (2005) and Saitz et al (2011). Although flaws can be noted in all the research and here, because the two groups contain different patients and the sample is not case controlled, this might be felt to damage any claims made, the results are too startling to be the result of coincidence. 


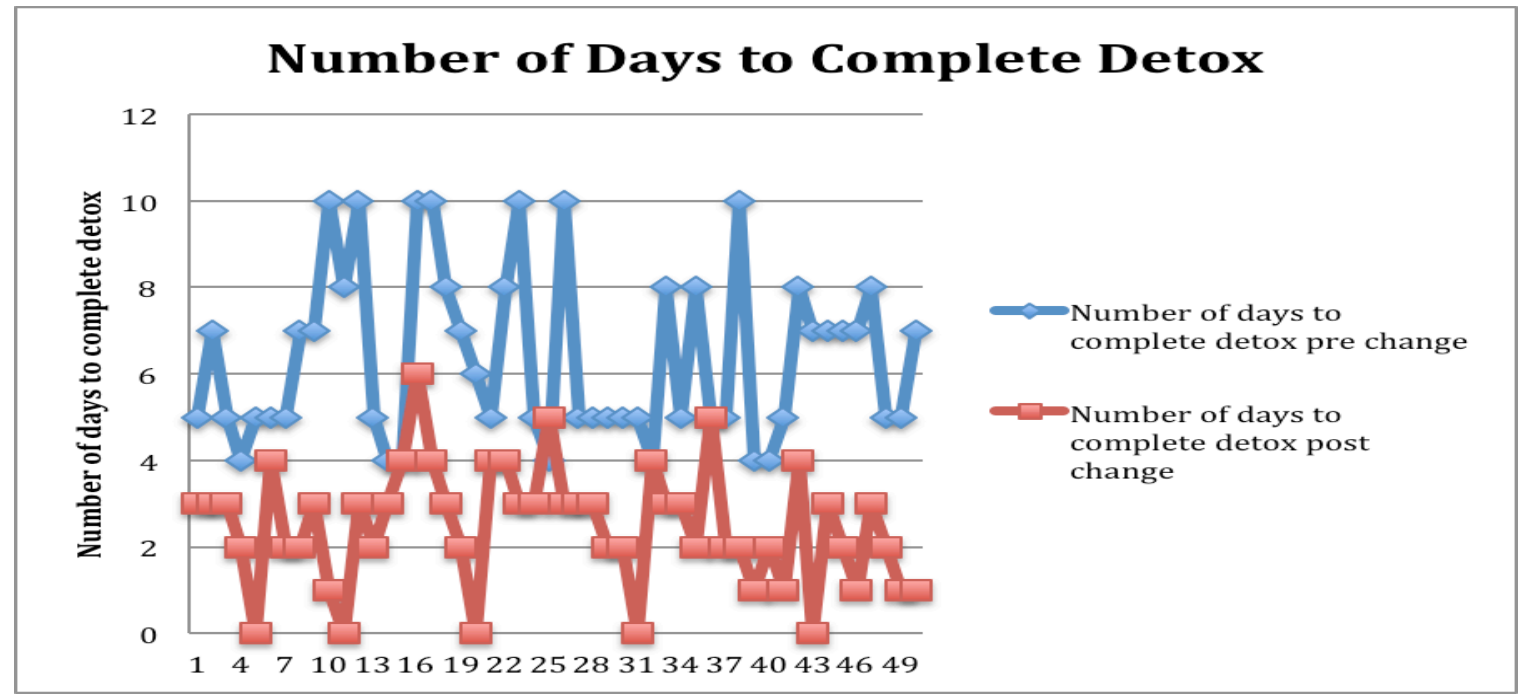

The comparitive graph is a clear indicator that the time for a patient to complete a detox has greatly been reduced since the implemetnation of the ICP. Furthermore, the amount of Chlordiazepoxide to succesfully complete a detox was also shown to decrease significantly.

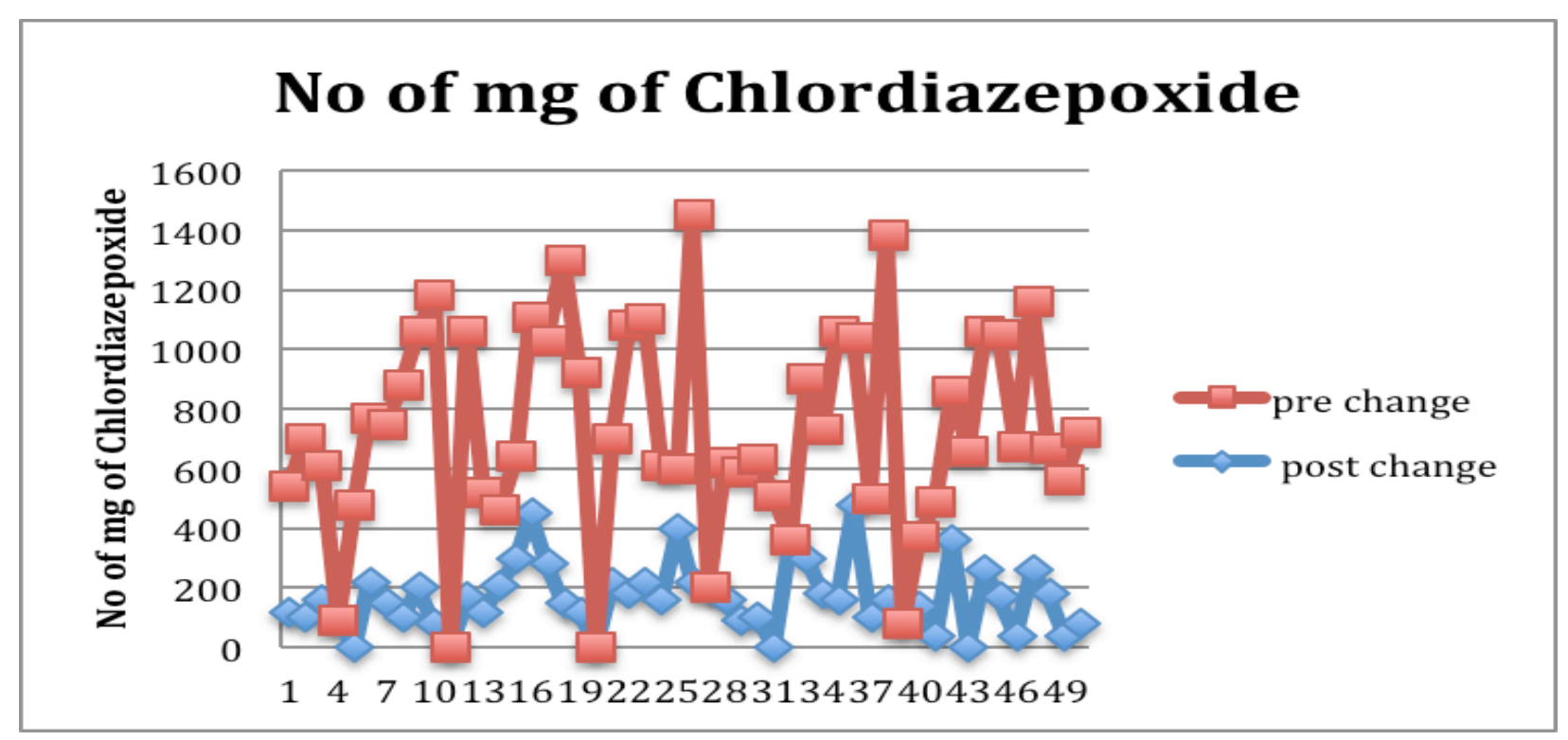

Even though less Chlordiazepoxide was required in the majority of cases to manage AWS, not one person from this sample group developed severe signs of withdrawal. It is most likely that this is due to the patients being assessed more frequently using a validated assessment tool in conjunction with staff having an increased knowledge of the withdrawal process tha before the introduction of the ICP. The fact that signs and symptoms were being detected earlier, ensured patients were adequetly 
medicated and autonomic responses were kept to a minimum safeguarding against the development of severe AWS.

It was clear that the ICP as a contained booklet was allowing staff to ensure documentation was correct it was acting as a guide to guarantee staff knew exactly when to assess and how to manage the level of withdrawal they were presented with.

If these results are translated into even very rough costings, the savings to the Trust are obvious. Pre implementation, the average cost of an inpatient stay for detoxification was $f 1,908$. Post implementation this reduced to $f 744$. More importantly, less drugs were used, appropriate drugs were used in all cases and no severe symptoms displayed by any of the patients from the audit sample, which was, as in the pre implementation audit, a set of 50 consecutive referrals to the service.

Implementation was not without its problems however and a key stage was the entry of new junior doctors to the Trust which really move the change forward. The policy was not a change in practice for these doctors who accepted it as Trust policy and began to use it, thus, to an extent, forcing the rest of the clinical team to use the ICP and move practice and the care of this patient group, forward.

For the point of view of the alcohol team who had spend an huge amount of time and energy educating and implementing the policy, the surge in referrals was a validation of their efforts. With the increase in compliance came the increase of support from alcohol services, with the team's early input and support ensured that a full alcohol history was obtained and motivation assessed leading to prompter discharges or more successful detox attempts. The alcohol team were also identifying trends it was noted early on that not all patients who are alcohol dependent show actual physical signs of withdrawal and require no medication to stop. Before moving to the symptom triggered approach to management patients would have automatically been commenced on a prophylactic detox regime and could be in hospital for up to ten days to complete it even though their bodies did not require any medication. Some people were completing detox in as little as two days. Both of these last points 
were showing nurses that if looked after appropriately the patients in the long run required less management and spent less time in hospital. The alcohol team were also having fewer complaints from their patients with regards to treatment. Due to their withdrawals being managed appropriately and being referred to the alcohol team promptly showed that the hospital was trying to help them, their stay was much more than just having pills four times a day they had proper treatment plans and aftercare organised.

The team's credibility was growing in the hospital and with that acceptance of the change followed. Clinicians realised that the ICP was indeed improving care, improving documentation, improving patient satisfaction and reducing clinical incidents and the benefits of its use became clear, backed up by the second audit results.

\section{Conclusion}

This project has achieved what it set out to achieve; an evidence based integrated car pathway for a group of patients for whom care had been less than optimum, resulting in repeated detoxification and poor outcomes. The benefits of the cost saving, both in terms of inpatient stay and drug use are quantifiable, the potential benefits of less repeat episodes of detoxification and the revolving door effect, as well as the greater patient satisfaction are less easy to quantity but this remains an aim for future work.

Excessive drinking is currently the second greatest risk to public health in developed countries (Kaner 2010). Although most of that risk is avoidable the risk is clearly apparent with the large number of hospital admissions every year, with as many as one in three attendees having consumed alcohol immediately before their presentation and more than two-thirds of attendances after midnight may be alcohol related (Crawford et al 2004). The emphasis on alcohol is becoming more apparent and with initiatives such as Commissioning for Quality and Innovation (CQUIN) payment framework enables commissioners to reward excellence by, linking a proportion of healthcare income to the achievement of the local quality improvement goals. The fact that alcohol is such a major problem and is linked to 
payments to Trusts makes it even more imperative that treatment is timely and effective and this project demonstrates one such method. 